\title{
Vibratory Residual Stress Relieving- A Review
}

\author{
S. N. Shaikh \\ (Department of Mechanical Engineering, M.E.S.College of Engineering, S.P. Pune University, Pune, India)
}

\begin{abstract}
All manufacturing and fabricating processes such as casting, welding, machining, molding, heat treatment, plastic deformation during bending, rolling or forging introduce residual stresses into the manufactured object. They are caused by rapid, unequal cooling as opposed to the stresses caused by external loading. These stresses affect fatigue life and dimensional stability of material. Diffèrent destructive and non destructive techniques are used to relieve these undesirable residual stressess such as thermal stress relief method. Thermal stress relief methods have limitations like economic and technical infeasibility. To overcome the limitations of these methods in past few decades various methods have developed like shot peening, Vibratory Stress Relief. In this paper different methods of vibratory stress relief are reviewed.
\end{abstract}

Keywords - Residual stress, SubHarmonic VSR, Vibratory stress relief,

\section{INTRODUCTION}

Residual stresses reside within the metal work piece. They are caused by rapid, unequal cooling as opposed to the stresses caused by external loading. Residual stress could be caused by localized yielding of the material, because of a sharp notch or from certain surface treatments like shot peening or surface hardening. Among the factors that are known to cause residual stresses are the developments of deformation gradients in various sections of the piece by the development of thermal gradients, volumetric changes arising during solidification or from solid state transformations, and from the differences in the coefficient of thermal expansion in pieces made from different materials. Residual stresses induced in a body after welding may lead to dimensional instability and it reduces strength and fatigue life of component that is why it is important to measure and check these residual stresses. To reduce residual stresses different stress relieving methods are used like vibratory stress relief techniques, shot peening and mechanical tumbling. To check the effectiveness of stress relief methods measurement of these stresses before and after the treatment is necessary. For that purpose different measurement techniques are used like mechanical, X-Ray, ultrasonic, and magneto-elastic methods. In this paper latest vibratory stress relief techniques are discussed and their relative advantages and limitations against other stress relief methods are studied.

\section{TECHNIQUES OF REDUCING RESIDUAL STRESSES}

In industries diffèrent destructive and non destructive techniques are used to relieve the undesirable residual stressess such as thermal stress relief, shot peening, Vibratory Stress Relief (VSR).

\subsection{Heat Treatment(Furnace methods)}

It is the well-known method which is used to relieve internal stresses. Thermal Stress Relieving is generally applied to metallic materials that have been cold-worked, formed, machined, flame-cut, or weldfabricated to reduce residual stresses for dimensional stability or reduced risk of premature failure in service. In this traditional method, the part is heated under carefully controlled conditions to several hundred degrees, maintaining this state till majority of the stresses have been relieved. It is then followed by controlled cooling to prevent introduction of new residual stresses. In many cases, this process is reiterated many times during the manufacturing process before the part is ready for use. During this process, the yield point of the material is substantially lowered, which allows for plastic flow in the material. This plastic flow in the material, consequently, causes redistribution and subsequent reduction of internal residual stresses. Although thermal methods have been widely used in industry for the past century, they have their own limitations in certain situations. In some cases, the cost of treatment of the part is very high in terms of energy and capital required. Additionally, oxide scales on the surface are formed that require subsequent finishing processes.

\subsection{Shot peening}

Shot peening is a cold working process used to increase the fatigue properties of metal components. During the peening process, compressive residual stresses are induced in the work piece by bombarding it with small spherical media called shot. It is a surface cold worked process used to minimize stress corrosion cracking and other modes of failure.Excessive peening can result in formation of many small cracks in the component. 
2.3 Vibratory stress relieving

Vibration, in its various forms, has been used to stabilize and to reduce distortion of parts. In this method workpiece is subjected to vibrations at a particular frequency and amplitude for a pre-determined time interval. The combination of work piece's internal stresses and this externally applied force causes it to cross its yield point, which results in plastic flow. This leads to redistribution and reduction of residual stresses. This technique has several advantages such as it can handle large components in a timely and cost effective manner. The actual process of vibratory stress relief is simple. It is the process of inducing a metal structure to be stabilized into one or more resonant or sub-resonant vibratory states. For that purpose high force exciters are used. Vibrational stress relief equipment consists of components like a vibrator, power supply and control equipment.

\section{HISTORY OF VSR METHOD}

Vibratory stress relief is considered as a new technology but history of which can be traced back to the late 1930's. During World War 2 it was first developed as a fatigue test and was used by both the German Air force and the US Navy as a means of testing aircraft wings and boat hulls. Hammer relaxation techniques were used to relieve stresses in metal pieces, in which repeated hammer blows were applied to produce high vibrations.

In 1943 R.T.McGoldrick and H.E.Saunders[1] concluded that to relieve stresses in steel castings and weldments vibration treatment is more beneficial than other methods like annealing process. They also recommened resonant vibration method. In 1980. Dawson and Moffar[2] successfully used vibrations and showed that $90 \%$ stresses are relieved from the sample of three different alloys. In 1995 C.A.Walker[3] by inducing vibrations into the low alloy steel showed stress relaxation $40 \%$.Yang developed finite element model to simulate vibratory stress relief after welding and to determine most effective vibration frequency choice i.e Resonant or Non Resonant. Rao[4] by applying vibration treatment to Rails and noted that after vibration treatment the resonance peak increased and resonance frequency decreased.

$\mathrm{S}$ Aoki[5], have proposed a method for reduction of residual stress using vibration during welding and also shown the effectiveness of the method. The effectiveness of the proposed method is examined in a probabilistic manner. Thick plates are used as specimens and ultrasonic vibration and low frequency vibration are applied. Some specimens of same size and material are welded using vibration during welding under the same conditions of amplitudes or frequencies. Reduction probabilities of residual stress are obtained from the expected values and the standard deviations of residual stresses assuming the normal distribution.

From the Research it is seen that furnace treatment has many disadvantages. It is expensive because large furnaces are available in the major centers which results in high transportation cost, high energy consumption and pollution is there due to furnace's method improves work piece's ability to undergo loading and fatigue life. Using VSR method we can stress relieved large components without sending them to a furnace. It also provides reduced energy consumption and pollution. In this method extra work which is needed for work piece surface preparation is not required. Due to these reasons in Industries many manufacturers are avoiding these methods on large parts and using VSR methods.

\section{DIFFERENT VSR TECHNIQUES}

Vibratory stress relief is a nonthermal stress relief method which is most commonly used to obtain dimensional stability in welded and cast fabrications. The precise amount of residual stress reduction depends on the type of product, material and equipment used which difficult to predict. Because of difficulties in measuring residual stresses and due to unknown effects of vibration the question of optimization of VSR has remained unanswered. Some researchers have suggested resonant frequencies and sub-Resonant frequencies. All these theories are based on vibrational amplitude that can be obtained in the workpiece .The actual process of vibratory stress relief is simple Depending on the size of component different setups are used to impart vibrations. It is the process of inducing a metal structure to be stabilized into one or more resonant or subresonant vibratory states. For that purpose high force exciters are used.Vibrational stress relief equipment consists of components like a vibrator, power supply and control equipment. Three different types of VSR approaches which are commonly used are Resonant VSR, Sub Resonant VSR and Sub-Harmonic [6]. Which are as follows, 


\subsection{Resonant VSR}

This process is achieved by vibrating the part to its natural frequency and large changing loads. To achieve optimal results, constant high cyclic amplitude and accurate frequency should stimulate the cyclic properties of the component.To get higher number of loading patterns, vibrators with high frequency ranges should be used. For better results it should be applied after rough machining processes because it reduces stresses due to machining as well. In cases where thermal methods fail to get required accuracy and stability, R-VSR can be applied just before finishing operations to achieve total stability. Researchers have shown resonant treatment to be the most effective treatment when the foregoing considerations have been taken into Account. High force exciters with stable characteristics at high speed are required for complex parts to accomplish this process.

\subsection{Modal Sub Resonant VSR}

In Resonant VSR if equipment is not capable of reaching resonant frequency of the part. Then sub Resonant vibratory stress relief method is used. In this method only the base of the peak is achievable. This is due to the peak being just out of range of the equipment used. It is called as Modal-Sub Resonant VSR since the mode shape is evident, but the peak is not achievable.In this method like Resonant VSR, better the frequency range of the equipment, better the process results

\subsection{Sub-harmonic VSR}

It was developed to deal with inconsistent results of different resonant vibration approach. This method has two principles.In this method subharmonic energy is used for the stress relief. After performing this process the harmonic curve of a thermally stressed metal part shifts and stabilizes to a new frequency location as it reduces thermal stress.In this method there is the cyclic loading of the part at frequencies below the natural frequency range.Former vibration methods are operated at the highest amplitude (resonance) that the workpiece experienced which leads to inconsistent results.It could damage the metal parts if maintained for an extended period of time. In this process metal components exhibit a harmonic reaction to induced energy. The harmonic curve occurs when the vibrated component cannot dissipate any more energy from the force inducers and responds with an out-of-proportion amplitude movement. This method is advancement over former vibration stress relief methods.Sub-harmonic process can be used during welding to reduce distortion from welding and cracking.

\section{CONCLUSIONS}

Many industries are using Vibratory stress relief methods world-wide for several decades to reduce stresses which shows acceptance as an alternative to thermal stress relief to achieve dimensional stability in welded and cast fabrications. There are many advantages of VSR method as compared to traditional methods i.e. Heat treatment of metal parts which are time and cost savings. This method can be used for that application which does not require change in crystal structure of the part. It has proved valuable on the components such as crushers, vibrating screens, feeder pans to reduce stress. Continuous work is required to explore the scope of this method and to become a widespread practice in the industry. Experimentation and research is required to check the effectiveness of this method.

\section{REFERENCES}

[1] R. T. McGoldrick, H.E. Saunders,Some Experiments In Stress Relieving Castings and welded structuresby Vibration,Journal of the American Society for Naval Engineers,Journal of the American Society for Naval Engineers, Volume-55,Issue-4,pages 589-609, November 1943.

[2] R. Dawnson, D.G.Moffat, 1980, Vibratory stress relief and fundamental study of its effectiveness, Journal of Engineering Materials and Technology 102(2):169-176.

[3] C.A.Walker,A.J. Waddell \& D.J.Johnstone.Vibratory stress relief An investigation of the underlying process.Proceedings Institute of Mechanical Engineers vol209, pp51-58 1995.

[4] D.Rao,L.Chen,Vibratory Stress Relief in manufacturing the Rails of Maglev system,Journal of Manufacturing science and Engineering,126,Issue-2,388-391,2004. 
[5] S.Aoki, Reduction of Residual Stress by Ultrasonic Surface Vibration, Japanese Society of Mechanical Engineers 1995.

[6] S.A. Raza Baqar1 , Y.R.Jain , P. Khanna,Vibratory Stress Relief Techniques: A Review of Present Trends and Future Prospects, International Journal of Emerging Technology and Advanced Engineering, Volume 4, Issue 11, November 2014.

[7] R. A.Claxton, Vibratory Stress Relieving- An effective alternative to thermal treatment for component Stabilization, Journal of heat treatment of metals, 1991.2 P.53-59.

[8] M.E.Robbins,Topics in vibratory stress relief of weldments,submitted to the faculty of Rensselaer Hartford December 2004.

[9] C. M.Adams, Bruce B. Klauba,Productive Applications of Mechanical Vibrations, Winter Meeting of ASME AMD-52,47-57,1982. 